conditions with proper follow-up than to take drugs that are prescribed 'off label'.

The benefits of having reliable evidence-based data that clinicians could use to provide safe treatments are immeasurable. The current situation, in which pregnant women suffer either by not receiving treatment or because they are prescribed analgesics, psychoactive medications, antimicrobials, diuretics, vaccines or other treatments that could harm them or their fetus, is unjust ${ }^{2,3}$. Correcting it should be a priority. Françoise Baylis is in the departments of bioethics, philosophy and obstetrics and gynaecology, Dalhousie University, Halifax, Nova Scotia B3H 3P7, Canada. e-mail: francoise.baylis@dal.ca
1. CIOMS International Ethical Guidelines for Biomedical Research Involving Human Subjects; available at http://www. cioms.ch/frame_guidelines_nov_2002.htm

2. Baylis, F. \& Kaposy, C. J. Obstet. Gynaecol. Can. 32, 473-476 (2010).

3. Lyerly, A. D., Little, M. O.\& Faden, R. Int. J. Fem. Approaches Bioeth. 1, 5-22 (2008)

See Editorial page 666, and comment online at go.nature.com/LqKQy2.

\title{
Males still dominate animal studies
}

\section{Many researchers avoid using female animals. Stringent measures should consign this prejudice to the past, argue Irving Zucker and Annaliese K. Beery, in the third piece of three on gender bias in biomedicine.}

$\mathrm{n}$ the 1990s, several surveys showed a significant sex bias in animal experiments in many biological disciplines, with researchers using a disproportionately high number of male animals. Given that animal models underpin the development of treatments for numerous diseases, this has serious implications for healthcare in women. So, to test whether or not the situation has improved, we recently conducted our own survey of almost 2,000 animal studies that were published in 2009 (ref. 1).

We found a male bias in 8 out of 10 biological disciplines, most pronounced in neuroscience ( 5.5 males to 1 female), pharmacology ( 5 males to 1 female) and physiology (3.7 males to 1 female). Although we identified a female bias in studies on reproduction and in the few immunology reports that indicated the animals' sex, $75 \%$ of studies in three highly cited immunology journals did not specify whether the animals used were male or female.

We also sampled the Thomson Reuters Web of Science database for 2009 to investigate the use of female mammals in animal studies for particular diseases, and then compared the results with the prevalence of those diseases in women worldwide (see graphic).

This revealed several alarming things. For example, diagnoses for anxiety and depression are more than twice as common in women than in men, but fewer than $45 \%$ of animal studies into these disorders apparently used females. Women have more strokes than men, with poorer functional outcomes, but only $38 \%$ of animal studies into strokes used females. Some thyroid diseases are seven to ten times more common in women, but only $52 \%$ of animal models used females. Other researchers have found that rodent studies into the effects of drugs on behaviour use males nearly exclusively, despite there being well-established differences in the ways men and women absorb and excrete drugs ${ }^{2}$.

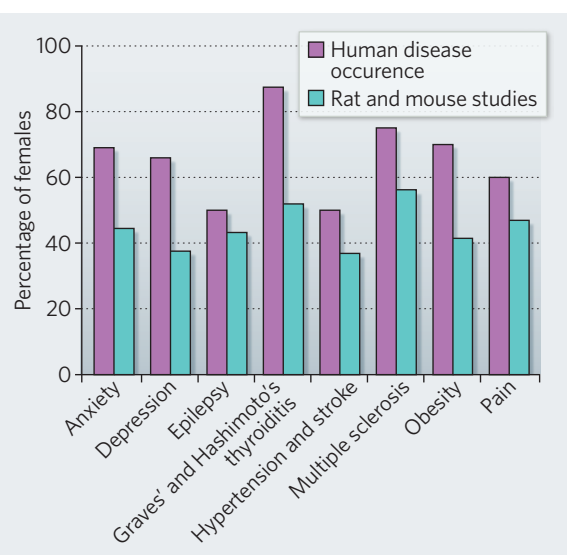

Gender gap. The percentage of women in the total population presenting with a disease (purple; see ref. 1) outstrips the percentage of females in rat and mouse models of that disease (green; data from Web of Science). Only studies with 'female' or 'male' as keywords were captured, so the chart underestimates male bias relative to a survey of individual articles by field.

The prejudice against using female animals may be partly due to concerns that they are intrinsically more variable than males because of cyclical reproductive hormones, making them unsuitable for use as baseline models. For instance, a 1923 study that showed marked oestrous-linked variations in movement-related activity in female rats ${ }^{3}$ may have discouraged the routine use of females in animal research. Yet there is little evidence to suggest that such variations make female animals inappropriate models. A 2005 meta-analysis found that female mice from many different strains were no more variable than males in the way they experienced pain. The researchers concluded that their findings "should force a reappraisal of the long-held assumption" that the oestrous cycle of female mice leads to greater variability in data ${ }^{4}$. Furthermore, hundreds of studies have shown that research using female animals is valid and reliable for numerous traits ${ }^{5,6}$. In research on human diseases such as epilepsy and multiple sclerosis, in which symptoms have long been known to be influenced by ovarian steroids, female animal models are de rigueur.

To correct the sex bias in animal research we need stringent, strictly enforced measures, not voluntary appeals. Journal editors and reviewers should require authors of research studies that use only male or only female animals to state this in the title of their papers. This would highlight sex biases and spur researchers to balance the numbers of males and females that they use. Funding agencies should refuse to consider grant proposals that do not properly acknowledge the sex of the animals to be used, and favour those that include males and females and plan to analyse data by sex.

We hope that changes such as these will make sex parity in animal research the norm. There are already some encouraging signs, such as the recent formation of the Organization for the Study of Sex Differences in Washington DC, and the announcement of a new journal, Biology of Sex Differences. It is time for researchers, editors and funding bodies to consign sexbiased animal studies to medical history.

Irving Zucker is in the Departments of Psychology and Integrative Biology, and the Helen Wills Neuroscience Institute, University of California, Berkeley, California 94720, USA. Annaliese K. Beery is at the Center for Health and Community, University of California, San Francisco, California 94143-0844, USA.

e-mail: irvzuck@berkeley.edu

1. Beery, A. K. \& Zucker, I. Sex bias in neuroscience and biomedical research. Neurosci. Biobehav. Rev. (in the press).

2. Hughes, R. N. Behav. Pharmacol. 18, 583-589 (2007)

3. Wang, G. H. Comp. Psychol. Monog. 6, 1-27 (1923).

4. Mogil, J. S. \& Chanda, M. L. Pain 117, 1-5 (2005).

5. Velísková, J. Neuroscientist 13,77-88 (2007).

6. Gold, S. M. \& Voskuhl, R. R. J. Neurol. Sci. 286, 99-103 (2009)

See Editorial page 666 , and comment online at go.nature.com/NJkvuJ. 